1 Oxford, UK

2 New York City, USA

3 New York City, USA

4 London, Canada

Correspondence to: S McPartlin sean.mcpartlin@1daysooner.org, C Weijer cweijer@uwo.ca Cite this as: BMJ 2020;371:m4258 http://dx.doi.org/10.1136/bmj.m4258 Published: 09 November 2020

\title{
CORONAVIRUS
}

\section{Covid-19 vaccines: Should we allow human challenge studies to infect healthy volunteers with SARS-CoV-2?}

The urgent need for covid-19 vaccines has prompted thousands of otherwise healthy people to volunteer to be infected with the virus to test candidate vaccines. Seán O'Neill McPartlin, Abie Rohrig, and Josh Morrison urge us to embrace the altruism of volunteers, but Charles Weijer argues that it would be dangerous and unjustified

Seán O’Neill McPartlin, ${ }^{1}$ Josh Morrison, ${ }^{2}$ Abie Rohrig, ${ }^{3}$ Charles Weijer ${ }^{4}$

Yes-Seán 0’Neill McPartlin, Josh Morrison, Abie Rohrig

Human challenge trials involve exposing healthy volunteers to a pathogen to learn more about the disease it causes and to test vaccines quickly. Much of our current understanding of coronaviruses comes from human challenge trials that researchers conducted in the 1960s, and efficacy data from such trials helped to license vaccines for cholera.

In the current pandemic, over 38 ooo people have signalled their willingness to participate in covid-19 human challenge studies with 1Day Sooner, a non-profit organisation advocating for these volunteers. The UK government has invested $£ 33.6 \mathrm{~m}$ (€37.3m; \$43.6m) to support such trials.

We see two main arguments for why these trials are valuable. First, while such trials are unlikely to accelerate the development of the first vaccines to hit the market, they may prove essential ${ }^{1}$ for second and third generation vaccines, which is essential, since rich countries are likely to outbid poorer ones for the first batch of vaccines.

There are over 300 candidate vaccines, and scientists will not be able to conduct conventional large scale phase III studies for all of them. Challenge studies can prioritise the best candidate vaccines to test and prove efficacy in cases where transmission is low. With a diverse portfolio of vaccines we are better positioned to optimise second, third, and nth generation vaccines for expeditious global distribution. Even if first generation vaccines arrive early this would still be sufficient reason to proceed with challenge studies. The moral importance of this should not be overlooked. Such studies are also the only efficient way to test prophylactic use of treatments such as monoclonal antibodies.

Second, challenge studies can help to answer essential questions about covid-19 immunity, such as correlates of protection ${ }^{2}$ and pathogenesis, which have a broad range of applications for vaccines and treatments. The instrumental benefits of such knowledge must be taken into account. ${ }^{3}$

It should not be assumed that we will have a licensed vaccine soon. A recent model reports a 50\% probability of no available vaccine being on the market by April 2021 and a 15\% chance of no vaccine by the end of 2021. To fail to prepare for such trials is a terrible gamble. Not only is it imprudent solely with respect to the odds but, importantly, it is immoral and unjust to gamble with the potential benefits at stake.

\section{Justifiable risks}

In The Ethics of Human Testing and the Coronavirus Vaccine, Weijer argues that other challenge studies were ethically permissible since the risk of incurable infection was reduced by the availability of a "rescue therapy." In other words, "this research was permissible because there are drugs that reliably cure both illnesses."4

This is not, and ought not to be, the standard of maximal risk that is morally permissible in human participant research. Rescue therapies for respiratory diseases are uncommon and do not exist for influenza or respiratory syncytial virus (RSV), two diseases for which challenge trials are often used. Neither a recent ethics overview of challenges published in Science ${ }^{5}$ nor the World Health Organization ${ }^{6}$ has endorsed the "rescue therapy" standard. Indeed, a fellow coauthor to the paper that Weijer cites does not agree with his analysis. ${ }^{7}$

A rescue therapy, in our view, is not an ethical prerequisite for a covid-19 challenge trial, as the risks of the disease in young healthy volunteers are on a par with or lower than other commonly accepted public service risks, even those in medical contexts. We allow ordinary members of society to risk exposure to covid-19, especially healthcare workers, many of whom lack personal protective equipment. We also let liver donors incur a 1 in 600 risk of death from donation ${ }^{8}$-orders of magnitude higher than the risk to young healthy volunteers in a covid-19 challenge trial, which would probably be less than 1 in $20000 .^{9}$

According to a principle of "risk parity," 10 if we allow some people to take certain risks to help save lives then we should allow other people to take similar, voluntary risks when there are comparable benefits. Put concretely: altruistic risks allowed in operating rooms (for example, kidney donation) should be allowed in clinical research facilities, such as in challenge trials. Any ethical oversight of such trials must, on this principle, consider the immense costs to ordinary people of blocking promising research. 
Volunteers are aware of the spectrum of risks facing challenge trial participants, and they will be subject to rigorous criteria to establish sound mental and physical health. ${ }^{11}$ Already, this is more than is expected in other analogous cases. Claims that such trials should not intentionally recruit people already at higher risk of contracting the virus (for example, healthcare workers or economically disadvantaged groups), so as to minimise the added risk of challenge trials, are well founded. Thankfully, this can be avoided in challenge trials, and thousands of informed volunteers are willing to take the risk so that others don't have to.

\section{No-Charles Weijer}

It would be unethical to allow healthy volunteers to be infected with wild-type SARS-CoV-2 in challenge studies to develop a vaccine. These challenge studies at present fail on ethical requirements of scientific and social value, reasonable benefits and risks, and just participant selection.

The scientific and social value of challenge studies is inadequate, as we are likely to have one or more vaccines in hand without them. Because of the risks of infecting healthy volunteers with a disease, challenge studies must demonstrate that the study's scientific ends could not be achieved by traditional means. Indeed, the core premise of SARS-CoV-2 challenge studies is that they will speed the development of a vaccine and thereby save "thousands or conceivably millions" of lives. ${ }^{12}$

Traditional vaccine research has proceeded with exceptional speed. As of 3 September 2020 there were 321 candidate vaccines for SARS-CoV-2. Of these, 33 are being tested in human clinical trials that plan to enrol 280 ooo people from 34 countries. We are told that "data to support licensure are anticipated to be available later this year."13

McPartlin and colleagues concede this point. They say, "While these trials are unlikely to accelerate the development of the first vaccines to hit the market, they may prove essential for second ... -generation vaccines." However, they do not provide a compelling account as to why developing second generation vaccines requires challenge studies. Plans to give poorer countries access to SARS-CoV-2 vaccines exist: for instance, the World Bank recently announced a €9.3bn (€10.3bn; \$12bn) programme "that will allow poor countries to purchase covid-19 vaccines to treat up to 2 billion people.”14

\section{Too much uncertainty}

The risks to participants in challenge studies are too uncertain and too great to be permitted. The ethics differ from those of patient clinical trials. Patients in the latter may be exposed to substantial risks provided that there is a reasonable prospect of direct benefit. But challenge studies make healthy people sick solely for scientific ends. Consequently, a more risk averse approach is the norm for challenge studies.

Thousands of volunteers have participated in challenge studies in the past 50 years, and none has died as a result. ${ }^{15}$ This is because scientists have steadfastly protected volunteers by restricting challenge studies to well understood diseases that would either resolve on their own and have no long term harmful effects or to diseases for which curative treatment is available.

McPartlin et al point to challenge studies with influenza and respiratory syncytial virus (RSV) in making the case for SARS-CoV-2 challenge studies. But SARS-CoV-2 is much less well understood than influenza or RSV. What we do know of the risks of SARS-CoV-2 infection suggests that they are too great to be permitted ethically. The manifestations of SARS-CoV-2 infection are protean and serious.
Beyond the lungs, the virus can infect the brain and cardiovascular system, resulting in stroke ${ }^{16}$ and myocarditis. ${ }^{17}$ And each of these sequelae has been observed in young people. Further, SARS-CoV-2 infection can be disabling, as demonstrated by people experiencing "long covid." 18

Finally, covid-19 is perhaps 10 times more lethal than influenza, and remdesivir, the drug to be made available to participants in the challenge trials, did not reduce mortality in the WHO Solidarity trial. ${ }^{19}$

\section{Unjust}

Strategies to mitigate research risks by selecting people at high risk of covid-19 for challenge studies are likely to be unjust. For example, advocates of SARS-CoV-2 challenge studies have proposed including people who are at high risk of the disease in the community. ${ }^{20}$ But this approach to participant selection may violate justice. It has become clear that some social groups, including Black, Asian, and minority ethnic groups, are at higher risk of the virus because of structural injustice. ${ }^{21}$ Selecting people from these groups for participation in challenge studies would compound unfairness and wrongly increase the burdens they face.

Bringing an end to the pandemic will require more than an effective vaccine: people must also be willing to take it. A recent global survey highlighted the problem of covid-19 vaccine hesitancy and found that the "accelerated pace of vaccine development has further heightened public anxieties and could compromise acceptance." 22 Public disclosure of serious injuries or deaths in SARS-CoV-2 challenge studies may undermine public trust and fuel vaccine hesitancy.

Traditional vaccine trials pose less risk to participants than challenge studies and, when combined with steps to enhance transparency, represent a safer path to vaccines that will be widely accepted.

\section{Biography}

Seán O'Neill McPartlin is a graduate student in philosophy at the University of Oxford. He is also a volunteer organiser of 1 Day Sooner and a cofounder of Effective Altruism Ireland, a community in Dublin organised around trying to do the most good.

Josh Morrison is the cofounder and executive director of 1 Day Sooner, a non-profit organisation that advocates on behalf of volunteers for a potential covid-19 human challenge trial. He previously cofounded and directed Waitlist Zero, a non-profit that advocates for living kidney donors, and the Rikers Debate Project, a non-profit that teaches competitive debating skills to students in Rikers Island jail in New York.

Abie Rohrig is the director of communications for 1 Day Sooner.

Charles Weijer is a bioethicist and an expert on the ethics of randomised controlled trials. This year he was a member of WHO's Working Group for Guidance on Human Challenge Studies in Covid-19.

Competing interests: We have read and understood BMJ policy and declare the following interests: CW receives consulting income from Cardialen, Eli Lilly \& Company, and Research Triangle Institute (RTI) International. SOM, JM, and AB have no competing interests to declare.

Provenance and peer review: Commissioned, not peer reviewed.

Banerjee S. Deliberately infecting volunteers with the coronavirus can answer vital questions about how it works. Telegraph2020 Sep 2. https://www.telegraph.co.uk/global-health/science and-disease/deliberately-infecting-volunteers-coronavirus-can-answer-vital/.

2 Haney DJ, Lock MD, SimonJK, Harris J, Gurwith M. Antibody-based correlates of protection against cholera analysis of a challenge study in a cholera-naiive population. Clin Vaccine Immuno/ 2017 (published online 31 May). https://www.ncbi.nlm.nih.gov/pmc/articles/PMC5583470/.

Askell A. In: Pummer T, Greaves H, eds. Evidence neutrality and the moral value of information in effective altruism: philosophical problems. Oxford University Press, 2020.

https://askell.io/publication/evidence-neutrality-and-the-moral-value-of-information. 
4 Weijer C. The ethics of human testing and the coronavirus vaccine. National Interest 2020 Sep 7. https://nationalinterest.org/blog/reboot/ethics-human-testing-and-coronavirus-vaccine-168449.

5 Shah SK, Miller FG, Darton TC, etal. Ethics of controlled human infection to address COVID-19. Science 2020;368:832-4. https://science.sciencemag.org/content/368/6493/832/tab-articleinfo. doi: 10.1126/science.abc1076 pmid: 32381590

6 World Health Organization. WHO Working Group for Guidance on Human Challenge Studies in Covid-19. Key criteria for the ethical acceptability of covid-19 human challenge studies. 2020 https://www.who.int/ethics/publications/key-criteria-ethical-acceptability-of-covid-19-humanchallenge/en/.

7 Savulescu J, Wilkinson D. Extreme altruism in a pandemic. J Med Ethics 2020 Apr 23 (blog). https://blogs.bmj.com/medical-ethics/2020/04/23/extreme-altruism-in-a-pandemic/.

8 Muzaale AD, Dagher NN, Montgomery RA, Taranto SE, McBride MA, Segev DL. Estimates of early death, acute liver failure, and long-term mortality among live liver donors. Gastroenterol 2020;142:273-80. https://pubmed.ncbi.nlm.nih.gov/22108193/.

9 Salje H, Tran Kiem C, Lefrancq N, etal. Estimating the burden of SARS-CoV-2 in France. Science 2020;369:208-11. https://science.sciencemag.org/content/early/2020/05/12/science.abc3517. doi: 10.1126/science.abc3517. pmid: 32404476

10 Chappell RY, Singer P. Pandemic ethics: the case for risky research. Res Ethics Rev 2020;16:1. https://journals.sagepub.com/doi/full/10.1177/1747016120931920doi:10.1177/1747016120931920.

11 Palmer A, Schuck C. 1Day Sooner Research Team. Establishing informed consent for SARS-CoV-2 challenge trials. Aug 2020. https://drive.google.com/file/d/1lsFSBB__CFLCSVMZDwB7s_dK28w6cwOW/view.

12 Eyal N, Lipsitch M, Smith PG. Human challenge studies to accelerate coronavirus vaccine licensure. J Infect Dis 2020;221:1752-6. https://academic.oup.com/jid/article/221/11/1752/5814216. doi: 10.1093/infdis/iiaa152 pmid: 32232474

13 Le TT, Cramer JP, Chen R, Mayhew S. Evolution of the COVID-19 vaccine development landscape. Nat Rev Drug Discov 2020;19:667-8. https:/www.nature.com/articles/d41573-020-00151-8. doi: 10.1038/d41573-020-00151-8. pmid: 32887942

14 Elliott L. World Bank announces $\$ 12$ bn plan for poor countries to buy covid vaccines. Guardian 2020 Sep 29. https://www.theguardian.com/business/2020/sep/29/world-bank-announcesplan-poor-countries-buy-covid-vaccines.

15 Evers DL, Fowler CB, Mason JT, Mimnall RK. Deliberate microbial infection research reveals limitations to current safety protections of healthy human subjects. Sci Eng Ethics 2015;21:1049-64. https://pubmed.ncbi.nlm.nih.gov/25150847/. doi: 10.1007/s11948-014-9579-z pmid: 25150847

16 Fifi JT, Mocco J. COVID-19 related stroke in young individuals. Lancet Neurol 2020;19:713-5. https://www.thelancet.com/journals/laneur/article/PIIS1474-4422(20)30272-6/fulltext. doi: 10.1016/S1474-4422(20)30272-6. pmid: 32822622

17 Hékimian G, Kerneis M, Zeitouni M, et al. Covid-19 acute myocarditis and multisystem inflammatory syndrome in adult intensive and cardiac care units. Chest 2020:S0012-3692(20)34359-2. https://www.sciencedirect.com/science/article/pii/S0012369220343592.

18 Greenhalgh T, Knight M, A'Court C, Buxton M, Husain L. Management of post-acute covid-19 in primary care. BMJ 2020;370:m3026. doi: 10.1136/bmj.m3026. pmid: 32784198

19 WHO Solidarity Trial Consortium. Repurposed antiviral drugs for covid-19-interim WHO Solidarity trial results.MedRxiv 2020 Oct 15. https://www.medrxiv.org/content/10.1101/2020.10.15.20209817v1.

20 Eyal N. Why challenge trials of SARS-COV-2 vaccines could be ethical despite risk of severe adverse events. Ethics Hum Res 2020;42:24-34. https://onlinelibrary.wiley.com/doi/full/10.1002/eahr.500056. doi: 10.1002/eahr.500056. pmid: 32441894

21 Trivedy C, Mills I, Dhanoya O. The impact of the risk of COVID-19 on Black, Asian and Minority Ethnic (BAME) members of the UK dental profession. Br Dent /2020;228:919-22. https://www.nature.com/articles/s41415-020-1781-6. doi: 10.1038/s41415-020-1781-6. pmid: 32591702

22 Lazarus JV, Ratzan SC, Palayew A, etal. A global survey of potential acceptance of a COVID-19 vaccine. Nat Med 2020. doi: 10.1038/s41591-020-1124-9. pmid: 33082575

This article is made freely available for use in accordance with BMJ's website terms and conditions for the duration of the covid-19 pandemic or until otherwise determined by BMJ. You may use, download and print the article for any lawful, non-commercial purpose (including text and data mining) provided that all copyright notices and trade marks are retained. 